survivors completed the survey. While more controls felt comfortable with their diet less than one month after operation ( $48.6 \%$ vs $41.3 \%)$, a greater proportion took over one year or never felt comfortable with their diet $(21.9 \%$ vs $18.0 \%)$. Ostomates had less problems with constipation $(P=0.001)$, and more with gas $(P=0.02)$ than controls. More cases avoided vegetables $(P=0.007)$, while a greater proportion of controls avoided carbonated beverages $(P<0.001)$ and utilized specialty diets $(P=0.03)$. There were multiple dietary and behavioral changes utilized to control bowel habits, including the timing of eating, exercise, and food avoidance. Conclusions: CRC survivors utilize multiple techniques to control their bowel function, and thus control their lives. Insight into these methods can lead to meaningful interventions for this population of cancer survivors.

\section{Abstract PS1-24 \\ Fatigue and Sleep Quality of Persons with Ostomies: \\ Gender Differences}

Mark C. Hornbrook, PhD, Center for Health Research, Kaiser Permanente Northwest; Carol M. Baldwin, PhD, RN, Arizona State University; Christopher Wendel, MS, Southern Arizona VA Health Care System; Marcia Grant, PhD, RN, City of Hope Medical Center; Lisa Herrinton, PhD, Kaiser Permanente Northern California; Jane Mohler, PhD, Arizona State University; Carmit McMullen, PhD, Kaiser Permanente Northwest; Robert S. Krouse, MD, Southern Arizona VA Health Care System

Background: Some colorectal cancer (CRC) patients require a permanent ostomy (a surgically created opening on the exterior of the abdomen that allows feces to be eliminated from the body). An ostomy can create psychosocial distress and affect body image, sense of self, and sexual functioning. Few studies have examined health-related quality of life (HR-QOL) relevant to disturbed sleep of CRC survivors with ostomies; moreover, no studies have examined gender differences of ostomates on quality of sleep and fatigue. Methods: HR-QOL was examined in this cross-sectional study of long-term ( $>5$ years) CRC survivors with ostomies, who receive care at Kaiser Permanente health systems in California, Oregon, Washington, and Hawaii. Participants completed the City of Hope Quality of Life Ostomy questionnaire, which includes demographic and scaled items, and narrative comments for greatest challenges associated with having an ostomy. Two items, 'fatigue' and 'sleep disruption' (scale from 0 to 10), served as dependent variables. Age, ethnicity, education, partnered status, body mass index (BMI), and time since surgery were included in models. Data were analyzed using t-tests and ordinal logistic regression modeling with significance set at $P<0.05$. Results: Women $(\mathrm{n}=118)$ compared to men $(\mathrm{n}=168)$ were less likely to be partnered $(25 \%$ vs $55 \%, P<0.0001)$. There were no differences for age, ethnicity, education, BMI, or time since surgery. Regression modeling for fatigue showed women to have lower HR-QOL compared to men $(0.65$ decrease, $P<0.01)$, adjusted for time since surgery (modest positive association, $P<0.05$ ). Regression modeling for sleep disruption also showed women to have poorer HR-QOL (0.57 decrease, $P<0.01$ ), adjusted for age (modest positive association, $P<0.001$ ). CRC surgical controls without ostomy showed no significant gender difference for either HR-QOL item. Qualitative narrative comments suggest sleep disruption is associated with fear of, or actual, leakage during hours of sleep. Conclusions: Women CRC survivors with ostomies report more fatigue and sleep disruption that may contribute to poorer HR-QOL compared to their male counterparts. Higher rates of fatigue for women are consistent with gender differences in other health conditions. These findings can provide a foundation for gender-specific ostomy interventions to improve sleep quality.

\section{Abstract PS1-25 \\ Statin Use is Associated With a Reduced Risk of Colon Cancer Recurrence}

Jody M. Jackson, RN, BSN, HealthPartners Research Foundation; Daniel M. Anderson, MD, MPH, Regions Hospital Cancer Center; Amy L. Butani, BA, HealthPartners Research Foundation; Stephen E. Asche, MA, HealthPartners Research Foundation; Cheri J. Rolnick, MPH, PhD, HealthPartners Research Foundation

Background: A significant body of data suggests that statin use reduces the risk of developing colon and other cancers, although other data have not confirmed these findings. Aims: To examine whether statin use helps prevent recurrence of colon cancer in patients with resected disease. Methods: We conducted a retrospective analysis of patients treated for stage I-III colon cancer in a large metropolitan health system. Incident cases of colon cancer between 1995, and the present were identified through the tumor registry of the health plan-owned hospital. All identified cases were then crosschecked against the HMO membership database. Data regarding statin use was pulled from the HMO outpatient pharmacy database. For each patient all available data from the 5-year window prior to first diagnosis of cancer until either recurrence or most recent data available was obtained. Demographic data and survival data from the tumor registry were then combined with pharmacy data regarding statin use in a single database for analysis. Recurrence and time to recurrent disease were compared in statin users and non-users. Results: A total of 358 colon cancer cases were available for analysis. Median follow-up of patients after diagnosis of colon cancer was 49 months. Eighty-nine patients $(24.9 \%)$ used statin drugs for some period of time with a median of 780 days of use. Median age of all patients enrolled was 69 years (range, 24-95 yrs). Statin use was higher in patients ages $61-75$ years $(33 \%)$ than in those aged $24-60$ years $(22 \%)$ or those aged $76-95$ years $(19 \%)$ $P=0.02$ ). There was no association between statin use and stage at diagnosis. Only 6 of 89 patients $(6.7 \%)$ with any statin use relapsed as opposed to 43 of $269(16 \%)$ of patients with no statin use (Fisher's exact, $P=0.03$ ). Kaplan-Meier analysis of relapse curves found a significant difference in relapse between groups with a 2-year recurrence rate of $3.7 \%$ among statin users and $10.1 \%$ among non-users ( $\log$ Rank, $P=0.03$, using age stratification). Conclusions: Statin use was associated with a decreased risk of recurrence in patients diagnosed with stages I-III colon cancer in this study. Further study of statin use in the prevention of colon cancer is warranted.

\section{Abstract PS1-26 \\ The Content of Physician-Patient Discussions During Routine Health Maintenance Exams}

Jennifer Elston Lafata, PhD, Henry Ford Health System; Greg Cooper, MD, University Hospitals of Cleveland, and Case Comprehensive Cancer Center; George Divine, PhD, Department of Biostatistics and Research Epidemiology, Henry Ford Health System; Susan Flocke, PhD, Departments of Family Medicine and Epidemiology and Biostatistics, Case Western Reserve University; Laura Siminoff, $\mathrm{PhD}$, Department of Behavioral Sciences and Health Promotion, Virginia Commonwealth University School of Public Health; Kurt Stange, MD, Departments of Family Medicine and Epidemiology and Biostatistics, Case Western Reserve University; Tracy Wunderlich, MA, Center for Health Services Research, Henry Ford Health System

Background: Little is known about the content of routine health maintenance exams (HMEs) delivered by primary care physicians (PCP) in today's busy primary care practices. We describe the frequency of patient-physician cancer screening discussions amidst the competing demands of other preventive services during routine HMEs to understand HME content in the real world of primary care. Methods: We use direct observation of PCP-patient interactions during HMEs to examine discussions around breast, colorectal (CRC), cervical, lung and prostate cancer screening and other lifestyle topics. Visits represent the first 103 visits completed in an NIH-funded study using in-office observations, audio-recordings and patient surveys to study CRC screening recommendations. Physician participants $(n=34)$ are general internists or family physicians practicing in a large integrated delivery system in southeast Michigan with patient-specific electronic medical record prompts for evidence-based cancer screening. Patient participants were aged 50-80 years who scheduled an HME with a participating physician and were due for CRC screening (i.e., no colonoscopy or barium enema within 10 years, flexible sigmoidoscopy within 5 years, or fecal occult blood testing (FOBT) within 12 months). Results: CRC screening was mentioned in $96 \%$ of observed HMEs. Among visits by women $(n=74), 85 \%$ mentioned breast and $51 \%$ cervical cancer screening. Among visits by men $(n=29), 72 \%$ mentioned prostate cancer screening. Lung cancer screening was mentioned in $15 \%$ of visits. The physician initiated these discussions more than $80 \%$ of the time, with the exception of lung cancer screening (53\%). Colonoscopy was overwhelmingly the procedure recommended for CRC screening $(82 \%)$, followed by FOBT (37\%) and others (4\%). Other topics frequently discussed were exercise $(88 \%)$, cholesterol $(81 \%)$, smoking $(68 \%)$, weight $(68 \%)$, blood pressure $(65 \%)$, and diet $(65 \%)$. While these discussions were usually initiated by physicians, patients were relatively more likely to initiate discussions around weight (41\%) and diet (35\%). Conclusions: In this study 